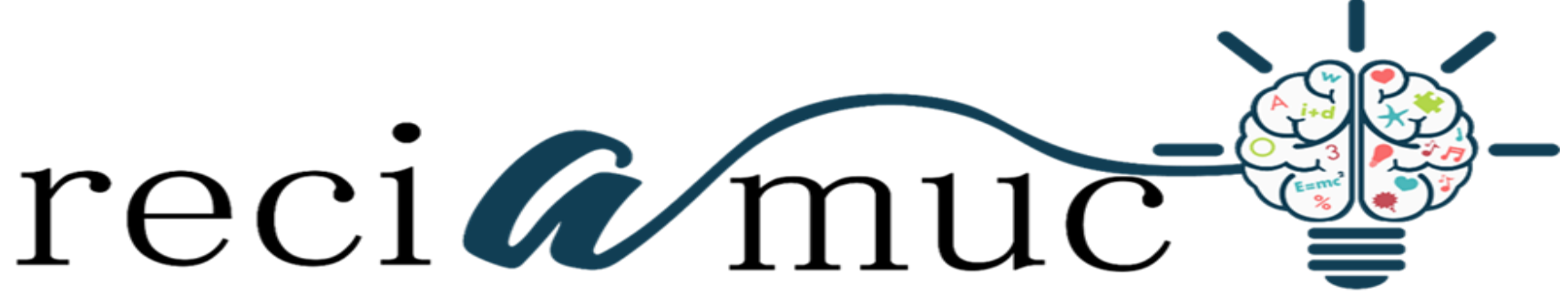

Revista cientifica de investigación actualización del mundo de las ciencias

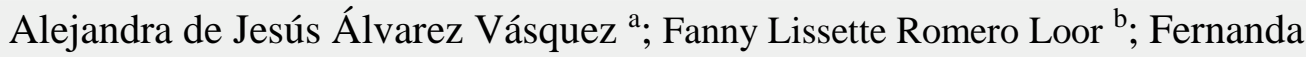
Gisell García Reyes ${ }^{\text {c; }}$ Mario Xavier Meza Cevallos ${ }^{\text {d; }}$ Karla Julen Vera Mendoza ${ }^{\mathrm{e}}$; Gema Margarita Reyes Ávila ${ }^{\mathrm{f}}$

Cuidados de anestesiología y reanimación para mantener las funciones vitales en pacientes donantes de órganos

Anesthesiology and resuscitation care to maintain vital functions in organ donor patients

Revista Científica de Investigación actualización del mundo de las Ciencias. Vol. 3 núm., 3, julio, ISSN: 2588-0748, 2018, pp. 1035-1064

DOI: $\underline{10.26820 / \text { reciamuc/3.(3).julio.2019.1035-1064 }}$

URL: http://reciamuc.com/index.php/RECIAMUC/article/view/314 Código UNESCO: 3205 Medicina Interna

Tipo de Investigación: Artículo de Revisión

(C) RECIAMUC; Editorial Saberes del Conocimiento, 2019

Recibido: $28 / 04 / 2019$

Aceptado: 19/05/2019

Publicado: 01/07/2019

Correspondencia: aleja alvarez14@hotmail.com

a. Médica General; Residente en Hospital San Vicente de Paul Ibarra; aleja alvarez14@ hotmail.com; Ibarra., Ecuador.

b. Médica Cirujana; Médico y cátedra de anatomía - Colegio Arcoíris; liss_frl@ hotmail.com; Portoviejo, Ecuador.

c. Médica Cirujana; Médico residente de anestesiología Hospital Gustavo Domínguez; fergigare@ gmail.com; Santo Domingo, Ecuador.

d. Médico Cirujano; Clínica Santa Margarita; xaviermxmc@gmail.com; Portoviejo, Ecuador.

e. Médico Cirujano; Médico General en funciones hospitalarias Hospital General del Norte de Guayaquil Los Ceibos (IESS); karlajvm@hotmail.com; Guayaquil, Ecuador.

f. Médico Cirujano; Médico General en funciones hospitalarias Hospital General del Norte de Guayaquil Los Ceibos (IESS); gemymargarita111@hotmail.com; Guayaquil, Ecuador. 


\section{Cuidados de anestesiología y reanimación para mantener las funciones vitales en pacientes donantes de órganos}

Vol. 3, núm. 3., (2019)

Alejandra de Jesús Álvarez Vásquez; Fanny Lissette Romero Loor; Fernanda Gisell García

Reyes; Mario Xavier Meza Cevallos; Karla Julen Vera Mendoza; Gema Margarita Reyes Ávila

\section{RESUMEN}

Aunque algunas publicaciones especifican las implicaciones anestésicas de cualquier muerte cerebral o muerte cardiaca, que carecen de detalles sobre la manera de proporcionar anestesia durante la cirugía de la donación de órganos. Se proporciona una descripción detallada de las consideraciones importantes anestésicas durante la cirugía de la donación de órganos en pacientes con cualquiera de estas dos muertes antes mencionadas. Una revisión exhaustiva de la literatura se llevó a cabo para localizar todos los artículos relevantes que describen los efectos sistémicos de cerebro y la muerte cardiaca y sus implicaciones anestésicas. Se requiere hallazgos principales altamente específicos en el manejo intraoperatorio por un anestesiólogo durante la obtención de órganos después de la muerte cerebral. Para gestionar el donante con muerte cerebral o cardiaca, el anestesiólogo debe incorporar el conocimiento de los efectos de la muerte cerebral en cada sistema de órganos, así como los efectos de las medidas preoperatorias que el donante se requiere en la unidad de cuidados intensivos. También es importante saber qué órganos van a ser adquiridos con el fin de establecer objetivos específicos y aplicar estrategias (por ejemplo, la ventilación pulmonar protectora o el control de la glucemia intraoperatoria) para optimizar los resultados de los donantes. Durante la obtención de órganos después de la muerte cardiaca, la participación directa de un anestesiólogo es particularmente importante para los donantes de pulmón.

Palabras Claves: Anestesia; Donantes de órganos; Procedimientos quirúrgicos; Cirugía. 


\title{
Cuidados de anestesiología y reanimación para mantener las funciones vitales \\ en pacientes donantes de órganos
}

Vol. 3, núm. 3., (2019)

Alejandra de Jesús Álvarez Vásquez; Fanny Lissette Romero Loor; Fernanda Gisell García Reyes; Mario Xavier Meza Cevallos; Karla Julen Vera Mendoza; Gema Margarita Reyes Ávila

\begin{abstract}
Although some publications specify the anesthetic implications of any brain death or cardiac death, they lack details on how to provide anesthesia during organ donation surgery. A detailed description of the important anesthetic considerations during organ donation surgery is provided in patients with either of these two aforementioned deaths. An exhaustive review of the literature was carried out to locate all the relevant articles describing the systemic effects of brain and cardiac death and their anesthetic implications. It requires highly specific main findings in intraoperative management by an anesthesiologist during organ procurement after brain death. To manage the donor with brain or cardiac death, the anesthesiologist must incorporate the knowledge of the effects of brain death on each organ system, as well as the effects of the preoperative measures that the donor is required in the intensive care unit. It is also important to know which organs are going to be acquired in order to set specific objectives and apply strategies (for example, protective lung ventilation or intraoperative blood glucose control) to optimize donor outcomes. During the procurement of organs after cardiac death, the direct participation of an anesthesiologist is particularly important for lung donors.
\end{abstract}

Key Words: Anesthesia; Organ donors; Surgical procedures; Surgery. 


\section{Cuidados de anestesiología y reanimación para mantener las funciones vitales en pacientes donantes de órganos}

Vol. 3, núm. 3., (2019)

Alejandra de Jesús Álvarez Vásquez; Fanny Lissette Romero Loor; Fernanda Gisell García

Reyes; Mario Xavier Meza Cevallos; Karla Julen Vera Mendoza; Gema Margarita Reyes Ávila

\section{Introducción.}

El número de pacientes en espera de trasplante de órganos es mucho mayor que el número de órganos sólidos disponibles (Steinbrook, 2007). En 2012, 15.967 trasplantes de riñón se realizaron en los Estados Unidos, mientras que 92,885 candidatos estaban en lista de espera activa para un trasplante de riñón; 5.209 pacientes murieron a la espera de un trasplante de riñón.Ese mismo año, 15.308 personas estaban en lista de espera de trasplante de hígado, 5.468 recibieron un trasplante de hígado, mientras que 2.187 pacientes murieron a la espera de un trasplante de hígado (U.S. Department of Health \& Human Resources, 2012). El uso de cada órgano donado disponible es crucial, ya que está maximizando su viabilidad.

Existe un grupo de literatura guía intensivistas en el manejo preoperatorio de donantes de órganos en la unidad de cuidados intensivos (UCI) en el establecimiento antes de la obtención de órganos. Por otra parte, se están realizando estudios para investigar los efectos de un enfoque basado en el protocolo de gestión de los donantes en las primeras horas después de la muerte cerebral.

La donación en personas con muerte cerebral son aquellos pacientes que han sufrido un daño neurológico terminal y han sido declarados con muerte cerebral. La Academia Americana de Neurología ha publicado criterios para la declaración de muerte cerebral (Wijdicks, Varelas, Gronseth, \& Greer, 2010) y cada institución debe tener sus propias políticas y procedimientos pertinentes que están en conformidad con las leyes locales. El Comité Ad Hoc de la Escuela de Medicina de Harvard para examinar la definición de muerte cerebral, emitió un informe que 


\section{Cuidados de anestesiología y reanimación para mantener las funciones vitales \\ en pacientes donantes de órganos}

Vol. 3, núm. 3., (2019)

Alejandra de Jesús Álvarez Vásquez; Fanny Lissette Romero Loor; Fernanda Gisell García Reyes; Mario Xavier Meza Cevallos; Karla Julen Vera Mendoza; Gema Margarita Reyes Ávila

esencialmente redefine la muerte, y, posteriormente, una definición legal formal de la muerte cerebral fue instituida en la década de 1970. Antes de esta redefinición significativa de la muerte, todos los trasplantes de órganos cadavéricos procedían de donantes a corazón latiente. Sin embargo, DBD condujo a la mejora de los resultados secundarios a la disminución de tiempo de isquemia relativa. Posteriormente, el número de donantes DBD se estabilizó, y el interés en el uso de donantes DCD ha sido recientemente renovado. El número de donantes DCD ha aumentado cada año durante la última década.

Una persona que ha sufrido la devastación neurológica y se sospecha que podría cumplir con los criterios de muerte cerebral debe ser identificada por su médico tratante como un potencial donante de órganos DBD. Su idoneidad como un donante a continuación, se puede evaluar sobre la base de la presencia de enfermedades médicas y comorbilidades. Si el paciente es considerado como un donante de órganos médicamente adecuado y es declarado con muerte cerebral, de acuerdo con las directrices institucionales y locales, se procede a obtener el consentimiento y la obtención de órganos. Tal un paciente se considera un donante de órganos utilizado si se trasplanta al menos un órgano procurado (Dominguez-Gil, Delmonico, \& Shaheen, 2011)

Para los donantes de DCD, la muerte se declara en función de criterios cardiopulmonares y se puede esperar que sea inminente debido a una enfermedad grave o accidente subyacente. El donante de DCD es un paciente que no cumple con los criterios estrictos para la muerte cerebral pero que sufrió lo que se considera una lesión cerebral no recuperable devastadora, y la familia ha decidido retirar la atención. La donación se considera a petición de la familia del paciente. Tras la retirada del soporte vital, el donante de DCD se declara muerto según los criterios 


\section{Cuidados de anestesiología y reanimación para mantener las funciones vitales en pacientes donantes de órganos}

Vol. 3, núm. 3., (2019)

Alejandra de Jesús Álvarez Vásquez; Fanny Lissette Romero Loor; Fernanda Gisell García

Reyes; Mario Xavier Meza Cevallos; Karla Julen Vera Mendoza; Gema Margarita Reyes Ávila

cardiopulmonares para la muerte. Normalmente, el posible donante de DCD puede tener una enfermedad neuromuscular en etapa terminal, una lesión neurológica grave o una enfermedad pulmonar terminal avanzada. Una vez que ha cesado la función cardiopulmonar, no se realizan esfuerzos de reanimación, y se permite que transcurra un período de cinco minutos antes de la obtención del órgano para garantizar la ausencia de auto reanimación. Al igual que con un donante DCD, el paciente se considera un donante de órganos utilizado si al menos un órgano obtenido es trasplantado.

En el camino del potencial al donante utilizado, pueden surgir numerosos factores para prevenir la utilización exitosa de los órganos. Estos factores de supervisión pueden ser fallas del sistema orgánico, características del donante u órgano, o una incapacidad para obtener el consentimiento adecuado para la donación de órganos, incluida la negativa expresada previa del paciente a ser un donante de órganos. Las contraindicaciones relativas a la utilización de órganos incluyen la edad avanzada del paciente, aunque no hay un corte firme, y el antígeno leucocitario humano (HLA) no coincide. Existe una variación significativa entre los centros, cirujanos, regiones y organizaciones de obtención de órganos sobre lo que constituye un donante aceptable para órganos individuales.

\section{Metodología.}

Para el desarrollo de este proceso investigativo, se plantea como metodología la encaminada hacia una orientación científica particular que se encuentra determinada por la necesidad de indagar en forma precisa y coherente una situación, en tal sentido (Davila, 2015) 


\section{Cuidados de anestesiología y reanimación para mantener las funciones vitales \\ en pacientes donantes de órganos}

Vol. 3, núm. 3., (2019)

Alejandra de Jesús Álvarez Vásquez; Fanny Lissette Romero Loor; Fernanda Gisell García Reyes; Mario Xavier Meza Cevallos; Karla Julen Vera Mendoza; Gema Margarita Reyes Ávila

define la metodología "como aquellos pasos previos que son seleccionados por el investigador para lograr resultados favorables que le ayuden a plantear nuevas ideas". (p.66)

Lo citado por el autor, lleva a entender que el desarrollo de la acción investigativa busca simplemente coordinar acciones enmarcadas en una revisión bibliográfica con el fin de complementar ideas previas relacionadas título del artículo que se está haciendo a través de una revisión de literatura, para así finalmente elaborar un cuerpo de consideraciones generales que ayuden a ampliar el interés propuesto.

Tipo de Investigación

Dentro de toda práctica investigativa, se precisan acciones de carácter metodológico mediante las cuales, se logra conocer y proyectar los eventos posibles que la determinan, así como las características que hacen del acto científico un proceso interactivo ajustado a una realidad posible de ser interpretada. En este sentido, se puede decir, que la presente investigación corresponde al tipo documental, definido por Castro (2016), "se ocupa del estudio de problemas planteados a nivel teórico, la información requerida para abordarlos se encuentra básicamente en materiales impresos, audiovisuales y /o electrónicos”. (p.41).

En consideración a esta definición, la orientación metodológica permitió la oportunidad de cumplir con una serie de actividades inherentes a la revisión y lectura de diversos documentos donde se encontraron ideas explicitas relacionadas con los tópicos encargados de identificar a cada característica insertada en el estudio. Por lo tanto, se realizaron continuas interpretaciones con el claro propósito de revisar aquellas apreciaciones o investigaciones propuestas por diferentes 


\section{Cuidados de anestesiología y reanimación para mantener las funciones vitales en pacientes donantes de órganos}

Vol. 3, núm. 3., (2019)

Alejandra de Jesús Álvarez Vásquez; Fanny Lissette Romero Loor; Fernanda Gisell García

Reyes; Mario Xavier Meza Cevallos; Karla Julen Vera Mendoza; Gema Margarita Reyes Ávila

investigadores relacionadas con el tema de interés, para luego dar la respectiva argumentación a

los planteamientos, en función a las necesidades encontradas en la indagación.

\section{Fuentes Documentales}

El análisis correspondiente a las características que predomina en el tema seleccionado, llevan a incluir diferentes fuentes documentales encargadas de darle el respectivo apoyo y en ese sentido cumplir con la valoración de los hechos a fin de generar nuevos criterios que sirven de referencia a otros procesos investigativos. Para (CASTRO, 2016) las fuentes documentales incorporadas en la investigación documental o bibliográfica, "representa la suma de materiales sistemáticos que son revisados en forma rigurosa y profunda para llegar a un análisis del fenómeno". (p.41). Por lo tanto, se procedió a cumplir con la realización de una lectura previa determinada para encontrar aquellos aspectos estrechamente vinculados con el tema, con el fin de explicar mediante un desarrollo las respectivas apreciaciones generales de importancia.

\section{Técnicas para la Recolección de la Información}

La conducción de la investigación para ser realizada en función a las particularidades que determinan a los estudios documentales, tiene como fin el desarrollo de un conjunto de acciones encargadas de llevar a la selección de técnicas estrechamente vinculadas con las características del estudio. En tal sentido, (Bolívar, 2015), refiere, que es "una técnica particular para aportar ayuda a los procedimientos de selección de las ideas primarias y secundarias”. (p. 71). 


\section{Cuidados de anestesiología y reanimación para mantener las funciones vitales \\ en pacientes donantes de órganos}

Vol. 3, núm. 3., (2019)

Alejandra de Jesús Álvarez Vásquez; Fanny Lissette Romero Loor; Fernanda Gisell García Reyes; Mario Xavier Meza Cevallos; Karla Julen Vera Mendoza; Gema Margarita Reyes Ávila

Por ello, se procedió a la utilización del subrayado, resúmenes, fichaje, como parte básica para la revisión y selección de los documentos que presentan el contenido teórico. Es decir, que mediante la aplicación de estas técnicas se pudo llegar a recoger informaciones en cuanto a la revisión bibliográfica de los diversos elementos encargados de orientar el proceso de investigación. Tal como lo expresa, (Bolívar, 2015) "las técnicas documentales proporcionan las herramientas esenciales y determinantes para responder a los objetivos formulados y llegar a resultados efectivos" (p. 58). Es decir, para responder con eficiencia a las necesidades investigativas, se introdujeron como técnica de recolección el método inductivo, que hizo posible llevar a cabo una valoración de los hechos de forma particular para llegar a la explicación desde una visión general.

Asimismo, se emplearon las técnicas de análisis de información para la realización de la investigación que fue ejecutada bajo la dinámica de aplicar diversos elementos encargados de determinar el camino a recorrer por el estudio, según, (Bolívar, 2015) las técnicas de procesamiento de datos en los estudios documentales "son las encargadas de ofrecer al investigador la visión o pasos que debe cumplir durante su ejercicio, cada una de ellas debe estar en correspondencia con el nivel a emplear" (p. 123). Esto indica, que para llevar a cabo el procesamiento de los datos obtenidos una vez aplicado las técnicas seleccionadas, tales como: fichas de resumen, textual, registros descriptivos entre otros, los mismos se deben ajustar al nivel que ha sido seleccionado.

Introducción. 


\section{Cuidados de anestesiología y reanimación para mantener las funciones vitales en pacientes donantes de órganos}

Vol. 3, núm. 3., (2019)

Alejandra de Jesús Álvarez Vásquez; Fanny Lissette Romero Loor; Fernanda Gisell García Reyes; Mario Xavier Meza Cevallos; Karla Julen Vera Mendoza; Gema Margarita Reyes Ávila

\section{Donación tras muerte cerebral}

La discusión de DBD comienza con el diagnóstico de muerte cerebral. Los médicos que discuten la donación de órganos con la familia del paciente deben ser diferentes de los que realizan la obtención del órgano. Antes de proceder a la obtención de órganos, el anestesiólogo debe asegurarse de que el diagnóstico de muerte cerebral se haya realizado de acuerdo con las pautas de la Academia Americana de Neurología. No es función del anestesiólogo realizar este diagnóstico.

El anestesiólogo desempeña un papel fundamental en la obtención de órganos del paciente con muerte cerebral, ya que la hemodinámica, la termorregulación, el estado del volumen intravascular y la parálisis del músculo esquelético requieren un manejo activo y son vitales para la obtención de órganos sanos. En presencia de muerte cerebral, la función de la médula espinal sigue intacta y los reflejos somáticos y viscerales permanecen (Conci, Procaccio, Arosio, \& Boselli, 1986). El manejo eficaz de la donación con anestesia requiere una comprensión de los efectos de la muerte cerebral en cada sistema de órganos.

Efectos de la muerte cerebral en sistemas de órganos y consideraciones anestésicas.

La causa de una lesión afecta a ciertos sistemas de órganos, pero casi todos los sistemas de órganos se ven afectados por la muerte cerebral. La literatura de cuidados críticos apoya la normalización de la fisiología del donante para maximizar la viabilidad a largo plazo de los órganos para la donación. Esta estrategia debe continuar desde la UCI al quirófano durante la cirugía de donación. 


\section{Cuidados de anestesiología y reanimación para mantener las funciones vitales \\ en pacientes donantes de órganos}

Vol. 3, núm. 3., (2019)

Alejandra de Jesús Álvarez Vásquez; Fanny Lissette Romero Loor; Fernanda Gisell García Reyes; Mario Xavier Meza Cevallos; Karla Julen Vera Mendoza; Gema Margarita Reyes Ávila

\section{Cardíaco}

La inestabilidad hemodinámica y la disfunción cardíaca tienen lugar después de la muerte cerebral. Si bien se han propuesto una serie de mecanismos para explicar estos cambios, la causa aún no se comprende por completo. Está claro que los factores neuronales y humorales desempeñan un papel, así como las condiciones de carga alteradas y la lesión miocárdica. Sin embargo, la opinión de algunos expertos es que la lesión miocárdica a largo plazo asociada con la muerte cerebral puede ser en gran medida una función de la disminución de la perfusión coronaria. Si bien la función cardíaca y el tono y el volumen intravascular parecen estar en una interacción complicada con la perfusión de la arteria coronaria después de la muerte cerebral, existe evidencia de que la función cardíaca se puede preservar si se mantiene la presión de perfusión coronaria (Szabo, 2004). Existe un acuerdo general de que, poco después de la muerte cerebral, un período prolongado de aumento del tono simpático y del gasto cardíaco se produce como resultado de una "tormenta de catecolaminas". Con este estallido de catecolaminas, la taquicardia y la vasoconstricción pueden causar hipertensión profunda. La lesión miocárdica asociada puede deberse a un aumento profundo en la resistencia vascular sistémica y ocasiona insuficiencia ventricular izquierda, disminución del gasto cardíaco e incluso insuficiencia de la válvula mitral. Pueden aparecer arritmias cardíacas junto con cambios electrocardiográficos indicativos de isquemia. Después de varias horas, puede producirse una pérdida dramática del tono simpático y resultar en una inestabilidad hemodinámica que puede comprometer la calidad de los órganos del donante (Belzberg, Shoemaker, \& Wo, 2007) Como se describe a continuación, esta pérdida del tono simpático debe ser gestionado en consecuencia. 


\section{Cuidados de anestesiología y reanimación para mantener las funciones vitales en pacientes donantes de órganos}

Vol. 3, núm. 3., (2019)

Alejandra de Jesús Álvarez Vásquez; Fanny Lissette Romero Loor; Fernanda Gisell García Reyes; Mario Xavier Meza Cevallos; Karla Julen Vera Mendoza; Gema Margarita Reyes Ávila

La hipovolemia puede exacerbar la inestabilidad hemodinámica. Hay múltiples contribuyentes a la pérdida de volumen en el paciente con muerte cerebral. Aunque la cirugía de obtención de órganos generalmente no es un procedimiento prolongado, pueden producirse cambios significativos de líquidos con una considerable acumulación intersticial de líquido debido a la incisión abdominal y / o torácica realizada para la obtención de órganos. También se producen algunas pérdidas por evaporación y pérdidas de sangre por incisión explícitas. La poliuria secundaria a diabetes insípida resultante de un infarto hipofisario posterior es común en la muerte cerebral y puede contribuir aún más a hipovolemia (Chen, Bittner, Kendall, \& Van Trigt, 1996). La estrategia de reemplazo de líquido intravascular varía para diferentes órganos; por lo tanto, se debe tener cuidado al tomar decisiones sobre el estado del volumen y la reanimación del donante. Por ejemplo, la administración moderada de líquido intravascular puede no tener efectos adversos en el hígado o los riñones, pero este líquido podría tener efectos nocivos en el pulmón o páncreas transplantadas. En consecuencia, algunos cirujanos consideran la administración intraoperatorios de líquido como el método preferido para mejorar la sangre, la presión en un paciente hipotensor con donante, con vasopresores relativamente contraindicados debido a la vasoconstricción y la disminución del flujo sanguíneo a órganos vitales. Sin embargo, los peligros para la función de los órganos a largo plazo derivados de la hipoperfusión de los órganos relacionada con la hipotensión y la reducción del suministro de oxígeno pueden superar los riesgos asociados con el uso de vasopresores. Por lo tanto, un plan equilibrado para mantener una presión arterial adecuada incorpora el reemplazo de los déficits de líquidos por vía intravenosa y, al mismo tiempo, utiliza vasopresores para contrarrestar la disminución de la resistencia vascular sistémica que se produce en los pacientes después de la muerte cerebral. Como se detalla más adelante, se ha demostrado 


\section{Cuidados de anestesiología y reanimación para mantener las funciones vitales \\ en pacientes donantes de órganos}

Vol. 3, núm. 3., (2019)

Alejandra de Jesús Álvarez Vásquez; Fanny Lissette Romero Loor; Fernanda Gisell García Reyes; Mario Xavier Meza Cevallos; Karla Julen Vera Mendoza; Gema Margarita Reyes Ávila

que el uso de vasopresina como parte de una estrategia de reemplazo hormonal aumenta la tasa de obtención exitosa de órganos mediante la restauración del tono vascular y la perfusión de órganos.

El manejo de los cambios cardiovasculares resultantes del aumento repentino de catecolamina después de la muerte cerebral aguda mejora la función sistólica y la posibilidad de un trasplante cardíaco exitoso (Audibert, Charpentier, \& Seguin-Devaux, 2006). Como se mencionó anteriormente, esta tormenta autónoma ocurre poco después de la muerte cerebral y generalmente dura solo varias horas. En consecuencia, no será un problema para el manejo en el quirófano durante la cirugía de donación de órganos; sin embargo, comúnmente se trata con nitroprusiato y / o esmolol. En la mayoría de los casos, los objetivos cardiovasculares deben incluir una presión arterial sistólica $[100 \mathrm{mmHg}$, una presión arterial media $[70 \mathrm{mmHg}$, con una frecuencia cardíaca de 60 a 120 latidos por minuto]. El primer paso en la reanimación cardiovascular debe ser el reemplazo efectivo del volumen intravascular. Más allá de la estrategia de transfusión de glóbulos rojos, que se describe a continuación, la elección de cristaloides o coloides puede guiarse por la planificación o no de la adquisición de pulmones. No se ha demostrado que ningún líquido en particular sea ventajoso, pero las soluciones de $\mathrm{NaCl}$ al $0,9 \%$ generalmente deben evitarse en grandes volúmenes para prevenir la acidosis hiperclorémica. Sin embargo, si se utiliza coloide como expansor de volumen intravascular, puede ser mejor evitar algunos de los productos de hidroxietil almidón (HES), ya que su uso en donantes con muerte cerebral se ha relacionado con la función de injerto renal inmediato deteriorada (Cittanova, y otros, 1996). 


\section{Cuidados de anestesiología y reanimación para mantener las funciones vitales en pacientes donantes de órganos}

Vol. 3, núm. 3., (2019)

Alejandra de Jesús Álvarez Vásquez; Fanny Lissette Romero Loor; Fernanda Gisell García Reyes; Mario Xavier Meza Cevallos; Karla Julen Vera Mendoza; Gema Margarita Reyes Ávila

Un estudio más reciente de una solución de HES con un peso molecular medio más bajo y una sustitución molecular mostró un impacto menor en la función del injerto renal inmediato ya largo plazo. Sin embargo, dado el potencial de insuficiencia renal duradera, habitualmente evitamos el uso de productos de HES en el cerebro de donantes muertos Si se planifica la obtención de pulmón, el agente preferido es el coloide. La reposición de volumen debe guiarse por la variación de la presión del pulso de la forma de onda arterial, la presión venosa central y la producción de orina si la diabetes insípida no es un problema. Algunos centros apuntan a una saturación venosa mixta de oxígeno de [60\%; sin embargo, hay una falta de evidencia de que esto influya en el resultado en términos de la cantidad de órganos trasplantados, injertos o receptores (Abdelnour \& Rieke, 2009). Es de suponer que este monitor se utiliza como un medio sustituto para garantizar la oxigenación apropiada del órgano y para garantizar que el suministro de oxígeno y consumo está adecuadamente equilibrado. Se puede usar soporte cardiovascular con dopamina o norepinefrina. Actualmente no hay evidencia de la superioridad de un agente sobre otro en el manejo del donante de órganos con muerte cerebral, aunque es prudente evitar grandes dosis de agonistas alfa-adrenérgicos para optimizar el flujo de sangre y el suministro de oxígeno a los órganos. La dopamina, la fenilefrina, la epinefrina, la norepinefrina y la vasopresina son agentes comunes y proporcionan un apoyo hemodinámico adecuado.

Algunos consideran que la vasopresina es el más ideal de estos agentes, ya que aborda la presión arterial y la diabetes insípida. Además, como se mencionó anteriormente, se ha demostrado que la vasopresina aumenta la tasa de recuperación del órgano tanto de manera independiente como parte de la terapia de reemplazo hormonal en donantes de órganos con muerte cerebral. La 


\section{Cuidados de anestesiología y reanimación para mantener las funciones vitales \\ en pacientes donantes de órganos}

Vol. 3, núm. 3., (2019)

Alejandra de Jesús Álvarez Vásquez; Fanny Lissette Romero Loor; Fernanda Gisell García Reyes; Mario Xavier Meza Cevallos; Karla Julen Vera Mendoza; Gema Margarita Reyes Ávila

infusión de vasopresina se dosifica en el rango de 0.01-0.04 UI min-1 en para minimizar las pérdidas de volumen de la diabetes insípida y para ayudar a la presión arterial. La dopamina, en dosis de $\backslash 5$ lg kg-1 min-1, mejora el flujo sanguíneo a los lechos vasculares renal, mesentérico y coronario, mejorando así la perfusión de órganos (Gelb \& Robertson, 1990), pero hay una falta de evidencia directa de que este efecto conduzca a una mejor supervivencia del injerto $\mathrm{u}$ otros resultados beneficiosos.

\section{Pulmonar}

La muerte cerebral puede llevar a un edema pulmonar neurogénico, ya que el aumento inicial de la resistencia vascular sistémica puede llevar a un aumento del volumen sanguíneo dentro del sistema venoso y la sobrecarga pulmonar posterior (Bratton \& Davis, 1997). El edema pulmonar también puede resultar de un gran volumen reanimación cristaloide. La presión hidrostática pulmonar elevada también puede dar lugar a edema pulmonar. Además, la liberación de catecolaminas durante el período hipertensivo e hiperdinámico inicial después de la muerte cerebral causa niveles elevados de citoquinas y, por lo tanto, daño endotelial pulmonar y alteración capilar. La dificultad respiratoria resultante puede progresar a apnea y paro cardíaco.

Se debe emplear una estrategia de ventilación con protección pulmonar, con volúmenes tidales de 6-8 $\mathrm{mL} / \mathrm{kg}$ y una presión espiratoria final positiva (PEEP) de $8-10 \mathrm{~cm}$, especialmente si se planifica la adquisición de pulmones. Se carece de evidencia para apoyar el mantenimiento de la alcalosis respiratoria durante la obtención de órganos. Si se planea la obtención de trasplantes de pulmón para el trasplante, se debe mantener un balance de líquidos mínimamente positivo. Se 


\section{Cuidados de anestesiología y reanimación para mantener las funciones vitales en pacientes donantes de órganos}

Vol. 3, núm. 3., (2019)

Alejandra de Jesús Álvarez Vásquez; Fanny Lissette Romero Loor; Fernanda Gisell García

Reyes; Mario Xavier Meza Cevallos; Karla Julen Vera Mendoza; Gema Margarita Reyes Ávila

ha demostrado que la terapia de reemplazo hormonal estandarizada (ver a continuación) junto con una cuidadosa atención para mantener la presión venosa central (CVP) \10 mmHg aumenta corazones y pulmones disponibles para trasplante sin disminuir la utilización de otros órganos.

\section{Endocrino}

La disfunción del eje hipotalámico-hipofisario ocurre en la mayoría de los pacientes con muerte cerebral y produce una disminución de las concentraciones séricas de las hormonas hipofisarias anteriores y / o posteriores (Novitzky, Cooper, \& Reichart, 1987). Estudios en animales han demostrado una reducción de triyodotironina (T3), cortisol e insulina después muerte cerebral.Un ensayo inicial en humanos proporcionó evidencia de que la terapia de pacientes con muerte cerebral con hormona tiroidea, cortisol e insulina mejoró tanto el estado cardiovascular como las tasas de trasplante de órganos secundarias a la mejora de la viabilidad de los órganos del donante. Aunque existe evidencia mixta, gran parte de esto sugiere que el tratamiento de donantes con muerte cerebral con una combinación de hormonas, como la hormona tiroidea, los glucocorticoides, la vasopresina y la insulina, aumenta las tasas de donación de órganos y puede mejorar la supervivencia del injerto y del paciente receptor. Muchos protocolos de tratamiento para donantes con muerte cerebral ahora incluyen la terapia con cada uno de estos medicamentos como práctica estándar. Este cóctel de hormonas se recomienda comúnmente para pacientes con muerte cerebral con una baja fracción de eyección del ventrículo izquierdo y se considera para todos los donantes (Tabla 1). Sin embargo, es necesario realizar estudios de dosis-respuesta para estas hormonas. En consecuencia, las dosis varían en diferentes estudios e instituciones, y hay poca evidencia para apoyar el uso de un protocolo sobre otro. Las recomendaciones en la Tabla 1 son 


\section{Cuidados de anestesiología y reanimación para mantener las funciones vitales \\ en pacientes donantes de órganos}

Vol. 3, núm. 3., (2019)

Alejandra de Jesús Álvarez Vásquez; Fanny Lissette Romero Loor; Fernanda Gisell García Reyes; Mario Xavier Meza Cevallos; Karla Julen Vera Mendoza; Gema Margarita Reyes Ávila

del Foro Canadiense sobre Gestión Médica para optimizar el potencial de los órganos de los donantes.

La evidencia de corticosteroides después del trasplante incluye ensayos controlados aleatorios y estudios observacionales. La mayoría de los ensayos controlados aleatorios que examinaron los corticosteroides los incluyeron en combinación con otros medicamentos, con efectos neutros. Los estudios observacionales generalmente muestran una mejor hemodinámica del donante, oxigenación, tasas de obtención de órganos, supervivencia del injerto y supervivencia del receptor.

Tabla 1. Terapia hormonal combinada recomendada para donantes de órganos con una fracción de eyección $\backslash 40 \%$ o inestabilidad hemodinámica y consideración en todos los donantes. (Shemie, Ross, \& Pagliarello, 2006)

\begin{tabular}{|c|c|}
\hline Hormona & Dosis \\
\hline $\begin{array}{c}\text { Hormona } \\
\text { tiroidea(tetraio } \\
\text { dothyronine) }\end{array}$ & $\begin{array}{c}20 \text { lg iv bolus, } 10 \lg \text { hr-1 } \\
\text { iv infusión }\end{array}$ \\
\hline Vasopressin & $\begin{array}{l}1 \text { Uivbolus, } 2.4 \mathrm{U} \mathrm{hr}^{-} \\
{ }_{1} \text { ivinfusion }\end{array}$ \\
\hline $\begin{array}{l}\text { Methylprednis } \\
\text { olone }\end{array}$ & $15 \mathrm{mg} \mathrm{kg}^{-1}$ iv q24hr \\
\hline
\end{tabular}

Donante con muerte cerebral el tratamiento es típicamente cortisol (3-5 mg kg-1 qd) versus metilprednisolona (15-60 mg kg-1 o 3-5 g una vez o qd). Una revisión retrospectiva de dosis altas de donantes con muerte cerebral (15 el tratamiento con corticosteroides con dosis bajas (300 mg de hidrocortisona) frente a dosis bajas (300 mg de hidrocortisona) encontró que el tratamiento con dosis bajas no produjo diferencias en la función pulmonar o cardíaca del donante, una tasa similar 


\section{Cuidados de anestesiología y reanimación para mantener las funciones vitales en pacientes donantes de órganos}

Vol. 3, núm. 3., (2019)

Alejandra de Jesús Álvarez Vásquez; Fanny Lissette Romero Loor; Fernanda Gisell García

Reyes; Mario Xavier Meza Cevallos; Karla Julen Vera Mendoza; Gema Margarita Reyes Ávila

de trasplante de órganos, un requerimiento de insulina disminuido y un mejor control glucémico.

(Dhar, Cotton, \& Coleman, 2013).

La muerte cerebral provoca trastornos hormonales que frecuentemente precipitan la hiperglucemia. La hiperglucemia puede exacerbarse por la liberación de epinefrina, la administración de esteroides exógenos o la infusión de soluciones que contienen dextrosa. La hiperglucemia se asocia con una menor tasa de aptitud del órgano para el trasplante y una menor supervivencia del injerto renal. El control de la glucosa sérica a niveles $\backslash 200 \mathrm{mg}$ dL-1, $11 \mathrm{mMol}$ L-1, se asocia con mejores resultados en pacientes con trasplante de páncreas. Este nivel debe mantenerse durante la obtención de órganos, especialmente si se planea un trasplante de páncreas. Se puede requerir una infusión de insulina, especialmente si se está infundiendo agua que contiene dextrosa para corregir la hipernatremia. Las recomendaciones varían, pero la mayoría de los expertos están de acuerdo en que se debe mantener la glucosa sérica $\backslash 150$ mg dL-1, 8 mMol L-1, aunque no está claro si existe alguna diferencia en las tasas de trasplante de órganos o la supervivencia del injerto siempre y cuando la glucosa sérica es $\backslash 180 \mathrm{mg}$ dL-1(Sally, Ewing, \& Crutchfield, 2014)

Como se mencionó, la muerte cerebral también puede alterar significativamente el eje de la hormona tiroidea, y el reemplazo de la hormona tiroidea también puede ser necesario con triyodotironina o una infusión de tiroxina para cumplir los objetivos hemodinámicos con un apoyo vasopresor mínimo. Estudios en animales con muerte cerebral demostraron que la función mejoró después de la suplementación hormonal con triyodotironina, cortisol e insulina. Informes posteriores en donantes humanos mostraron una función cardíaca mejorada con reemplazo de 


\section{Cuidados de anestesiología y reanimación para mantener las funciones vitales \\ en pacientes donantes de órganos}

Vol. 3, núm. 3., (2019)

Alejandra de Jesús Álvarez Vásquez; Fanny Lissette Romero Loor; Fernanda Gisell García Reyes; Mario Xavier Meza Cevallos; Karla Julen Vera Mendoza; Gema Margarita Reyes Ávila

hormona tiroidea. Sin embargo, un metaanálisis reciente de cuatro ensayos clínicos aleatorios que evaluaron el índice cardíaco en DBD mostró que la triyodotironina no mejoró la hemodinámica y sugiere que la triyodotironina no es la causa del miocardio Disfunción en pacientes con muerte cerebral. Con pruebas mixtas y un metanálisis que no muestra un beneficio claro de la administración de reemplazo de hormona tiroidea, algunos expertos sugieren que la terapia de reemplazo de tiroides no debe ser parte del manejo del donante con muerte cerebral a menos que el paciente tenga antecedentes de hipotiroidismo (Sally, Ewing, \& Crutchfield, 2014). Aun así, como se mencionó, los estudios han demostrado que la terapia de reemplazo hormonal de tres fármacos con triyodotironina / levotiroxina (T3 / T4), metilpred-nisolona y vasopresina aumenta el número de órganos viables para trasplante en un 20\% y mejora el injerto temprano función.

En consecuencia, muchos centros de trasplante administran rutinariamente terapia de reemplazo de tiroides en esta población de pacientes. Como parte de la terapia de reemplazo hormonal continuada y para ayudar al apoyo hemodinámico, se puede realizar una infusión de T3 / T4 en la sala de operaciones (triyodotironina (T3) 0.4 lg en bolo, 3 lg hr-1 infusión; levotiroxina (T4) 20 lg en bolo, 10 -50 lg hr-1 infusión) (Bratton \& Davis, 1997).

El infarto de la hipófisis posterior en el contexto de la muerte cerebral puede conducir a diabetes insípida, produciendo grandes cantidades de orina diluida y la hiperosmolaridad del suero resultante. La arginina vasopresina (AVP) debe utilizarse para reducir la producción de orina y evitar un aumento adicional de la osmolaridad sérica. Además, se ha demostrado que el uso de AVP en el donante con muerte cerebral aumenta la tasa de recuperación de órganos y la 


\section{Cuidados de anestesiología y reanimación para mantener las funciones vitales en pacientes donantes de órganos}

Vol. 3, núm. 3., (2019)

Alejandra de Jesús Álvarez Vásquez; Fanny Lissette Romero Loor; Fernanda Gisell García

Reyes; Mario Xavier Meza Cevallos; Karla Julen Vera Mendoza; Gema Margarita Reyes Ávila

supervivencia del injerto, al disminuir la hiperosmolaridad plasmática y mejorar la presión arterial sistémica, así como el gasto cardíaco. (Pennefather, Bullock, Mantle, \& Dark, 1995)

Es posible que se necesite un líquido hipotónico o una infusión de agua gratuita para corregir la hipernatremia. Los donantes con niveles de sodio en suero [155 mEq L-1 mostraron una tasa significativamente mayor de pérdida temprana del injerto, mientras que aquellos con niveles de sodio en suero corregidos no mostraron una tasa tan alta de pérdida temprana del injerto. La hipernatremia en la muerte cerebral debe considerarse una Estado hipovolémico. Si los niveles séricos de sodio persisten [155 mEq L-1 después de un volumen adecuado de reanimación, los autores sugieren la infusión de una solución hipotónica, como $\mathrm{NaCl}$ al 0,45\% (77 mEq L-1), como líquido de mantenimiento. En general, la corrección debe ocurrir a una tasa de 0.5-1.0 mEq L-1

hr-1 para evitar el edema cerebral y sus secuelas. Sin embargo, se desconoce si la corrección de la hipernatremia puede ocurrir a un ritmo más rápido en el paciente con muerte cerebral sin consecuencias negativas. El déficit hídrico se puede estimar utilizando los siguientes

\section{Musculo esquelético}

Incluso en el contexto de la muerte cerebral, pueden producirse movimientos somáticos. Los estudios en animales muestran que estos están mediados por reflejos de la médula espinal que se encuentran por debajo del nivel del tronco encefálico. Un estudio en animales demostró que la concentración alveolar mínima no fue alterada por la sección de la médula cervical alta y sugirió que los anestésicos inhiben la anestesia motora para la respuesta de la cirugía de donación de 


\section{Cuidados de anestesiología y reanimación para mantener las funciones vitales \\ en pacientes donantes de órganos}

Vol. 3, núm. 3., (2019)

Alejandra de Jesús Álvarez Vásquez; Fanny Lissette Romero Loor; Fernanda Gisell García Reyes; Mario Xavier Meza Cevallos; Karla Julen Vera Mendoza; Gema Margarita Reyes Ávila

órganos a través de los sitios en la médula espinal. Los movimientos somáticos son desinhibidos reflejos de los espines que ocurren estímulos como incisión quirúrgica o manipulación.

Debe proporcionarse parálisis del músculo esquelético durante la obtención del órgano para optimizar las condiciones quirúrgicas. Además, la relajación muscular mejorará la respuesta somática al estímulo quirúrgico mediado por los reflejos de la médula espinal, que pueden persistir incluso en presencia de muerte cerebral.

\section{Hematológica}

La incidencia informada de coagulopatía después de una lesión cerebral varía dramáticamente de $\backslash 10 \%$ a [80\%, aunque es probable que esta variabilidad se deba en parte a definiciones inconsistentes de coagulopatía y lesión cerebral.63 La coagulopatía puede ocurrir en una lesión craneal aislada y el desarrollo de coagulopatía después de que la lesión cerebral se asocie con un peor resultado.64 La liberación del factor tisular del parénquima cortical lesionado puede contribuir a, o incluso ser responsable de, el desarrollo de coagulopatía y, cuando es grave, la coagulación intravascular diseminada subsiguiente.64 La liberación de gangliósidos cerebrales y se cree que los sustratos ricos en plasminógeno del tejido cerebral dañado también contribuyen a la coagulopatía en pacientes con lesión cerebral grave.65 Por otro lado, en un estudio observacional prospectivo reciente de 345 pacientes con lesión cerebral traumática aislada grave, no hay asociación con el desarrollo de Se encontró coagulopatía.64

En Canadá, las recomendaciones para el manejo del paciente donante de órganos en la UCI incluyen apuntar a una concentración de hemoglobina de 9-10 g dL-1 y no menos de 7 g dL-137 


\section{Cuidados de anestesiología y reanimación para mantener las funciones vitales en pacientes donantes de órganos}

Vol. 3, núm. 3., (2019)

Alejandra de Jesús Álvarez Vásquez; Fanny Lissette Romero Loor; Fernanda Gisell García

Reyes; Mario Xavier Meza Cevallos; Karla Julen Vera Mendoza; Gema Margarita Reyes Ávila

para optimizar el suministro de oxígeno a los órganos. Las plaquetas y el plasma deben administrarse cuando sea evidente un sangrado clínicamente significativo, pero no para alcanzar un nivel de prueba de coagulación o un recuento plaquetario específico. No se cuenta con evidencia firme para estas recomendaciones en esta población de pacientes, pero la mayoría de los expertos y las pautas para el manejo de donantes con muerte cerebral sugieren objetivos de transfusión similares. Las recomendaciones para una concentración óptima de hemoglobina parecen basarse en la idea de que los pacientes críticamente enfermos a menudo tienen un mayor consumo global de oxígeno, pero también pueden tener una capacidad limitada para aumentar el suministro de oxígeno si la concentración de hemoglobina cae por debajo de algún umbral crítico.66 Además, algunas pautas sugieren apuntar a una saturación venosa mixta de oxígeno de C $60 \%$ para asegurar el suministro de oxígeno adecuado.37 En el caso de que alcanzar este objetivo sea un desafío, la transfusión de glóbulos rojos compactados puede mejorar la saturación venosa mixta de oxígeno.

\section{Consideraciones adicionales}

Antes del inicio de la cirugía de donación de órganos, deben colocarse catéteres intravenosos adecuados para el reemplazo rápido de líquido intravascular de gran volumen. Además, se recomienda la presencia de un catéter venoso central antes del inicio de la cirugía. Debe colocarse un catéter arterial para que la presión arterial pueda seguirse continuamente y manejarse intraoperatoriamente. Algunas pautas sugieren un catéter de la arteria pulmonar para el manejo de cuidados intensivos de estos pacientes con el fin de seguir el gasto cardíaco, la presión de enclavamiento capilar pulmonar y la resistencia vascular sistémica, pero faltan pruebas para demostrar que esto cambia el resultado. Además, es importante comunicarse con el equipo 


\section{Cuidados de anestesiología y reanimación para mantener las funciones vitales \\ en pacientes donantes de órganos}

Vol. 3, núm. 3., (2019)

Alejandra de Jesús Álvarez Vásquez; Fanny Lissette Romero Loor; Fernanda Gisell García Reyes; Mario Xavier Meza Cevallos; Karla Julen Vera Mendoza; Gema Margarita Reyes Ávila

quirúrgico y saber qué órganos se obtendrán para el trasplante ya que esto puede afectar el manejo anestésico. Como se describió anteriormente, si se planea la adquisición de pulmones, se debe usar una estrategia de ventilación con protección pulmonar, se debe mantener un balance de líquidos mínimamente positivo y se prefiere coloide sobre cristaloide para reemplazo de volumen. Antes de la heparinización, el equipo cardíaco puede solicitar la extracción de las líneas centrales permanentes o los catéteres de la arteria pulmonar que atraviesan la vena cava superior, ya que posteriormente sujetarán estos vasos antes de la extracción del corazón. Después de que el anestesiólogo administra heparina, los equipos quirúrgicos canalizan los principales vasos sanguíneos arteriales; el equipo cardíaco utiliza la aorta ascendente y el equipo abdominal utiliza la aorta abdominal infrarrenal

\section{Donación tras muerte cardíaca}

La donación de órganos de pacientes que no han sido declarados muerte cerebral está incluida en la categoría de DCD. Estos donantes se conocen como donantes no palpitantes (NHBD). Se declaran muertos según la definición cardiopulmonar después del cese de la atención de soporte vital en el contexto de lesiones neurológicas devastadoras y no reversibles, enfermedad pulmonar grave o enfermedad neuromuscular en etapa terminal (Bratton \& Davis, 1997) Cada hospital que sea un centro de trasplante o participe en La obtención de órganos debe tener un protocolo específico para DCD. El protocolo debe abordar los problemas de manejo preoperatorio de los donantes, consentimiento informado, retiro de medidas de soporte vital, pronunciamiento de muerte, recuperación de órganos y consideraciones financieras (Steinbrook, 2007). 


\section{Cuidados de anestesiología y reanimación para mantener las funciones vitales en pacientes donantes de órganos}

Vol. 3, núm. 3., (2019)

Alejandra de Jesús Álvarez Vásquez; Fanny Lissette Romero Loor; Fernanda Gisell García

Reyes; Mario Xavier Meza Cevallos; Karla Julen Vera Mendoza; Gema Margarita Reyes Ávila

En nuestra institución, la UCI o el personal del hospital realizan la cesación de la atención, incluida la extubación, con la presencia de un representante de la organización de obtención de órganos (OPO). El equipo de trasplante quirúrgico se mantiene en un lugar separado durante este proceso, a la espera de la progresión a la muerte cardíaca, mientras que el intensivista y el representante de la OPO continúan monitoreando y registrando los signos vitales después de la extubación. Si se logra la asistolia, hay un período de cinco minutos de observación adicional para asegurar que no haya auto-reanimación, momento en el cual el donante es declarado muerto por el personal del hospital. Durante estos cinco minutos, o inmediatamente después, el paciente es transportado a la sala de operaciones y colocado adecuadamente. En este momento, el equipo quirúrgico puede ingresar al quirófano y proceder con la obtención del órgano. Los miembros de la familia a menudo están presentes desde el momento de la extubación o el retiro de la atención, hasta el momento en que comienza la adquisición. Por lo tanto, se debe llevar a cabo una planificación adecuada para salir del área de la sala de operaciones antes de comenzar la adquisición. Si se van a adquirir los pulmones, un anestesiólogo reintubará la tráquea del paciente. La extubación traqueal no se realiza en todas las instituciones; más bien, el circuito del ventilador simplemente se desconecta del tubo endotraqueal (ETT). Además, en algunas instituciones, los pacientes están en la mesa de la sala de operaciones, preparados y cubiertos, antes de retirar la atención para minimizar el tiempo de isquemia caliente. En nuestra institución, el personal de cuidados críticos retira la atención en un área de espera inmediatamente adyacente a la sala de operaciones, y los familiares del paciente pueden pasar este tiempo con el paciente. Se utilizan límites de tiempo isquémicos cálidos para maximizar la idoneidad del órgano, ya que la hipotensión y el cese de la circulación conducirán a un suministro deficiente de oxígeno a los 


\section{Cuidados de anestesiología y reanimación para mantener las funciones vitales \\ en pacientes donantes de órganos}

Vol. 3, núm. 3., (2019)

Alejandra de Jesús Álvarez Vásquez; Fanny Lissette Romero Loor; Fernanda Gisell García Reyes; Mario Xavier Meza Cevallos; Karla Julen Vera Mendoza; Gema Margarita Reyes Ávila

órganos del donante y comprometerán la calidad del órgano del donante. Aunque los centros de trasplante pueden variar en su definición y duración del tiempo de isquemia, por lo general, el tiempo comienza con la extubación / desconexión de la ETT y termina con la perfusión de órganos con una solución de conservación en frío. El tiempo máximo permitido para la retirada de la atención, incluida la extubación, hasta la asistolia es diferente para los diferentes órganos. Si la asistolia no se produce dentro de este período de tiempo, esos órganos no se extraen. Ese tiempo es de 30 minutos para el pulmón, el hígado y el páncreas, y 60 minutos para el riñón. El corazón y el intestino no son candidatos para el trasplante de un donante de DCD, aunque el uso de cardioplejía puede ofrecer cierta promesa para el uso del corazón de un donante de DCD. El orden de obtención de órganos durante DCD es pulmones, hígado, páncreas, riñones.

\section{El papel de los anestesiólogos en la obtención de órganos DCD}

A diferencia de los médicos de cuidados críticos, los anestesiólogos no tienen capacitación sobre el final de la vida como parte de su plan de estudios básico. La transferencia completa de la atención a un anestesiólogo para las etapas terminales de la atención corre el riesgo de violar un principio básico de la medicina paliativa, es decir, los pacientes merecen la continuidad de la atención y proveedores constantes durante todo el proceso de muerte(RD., 2003) En consecuencia, el equipo de atención crítica puede participar directamente en la etapas finales de la vida durante el proceso de DCD, aunque un anestesiólogo puede proporcionar parte de la atención en el quirófano. Tras el cese de la ventilación mecánica y la terapia con vasopresores, el médico de la UCI que atiende al paciente puede dirigir la administración de opioides y benzodiacepinas ajustadas a la comodidad del paciente. A menudo, un opioide y una benzodiazepina se titulan con 


\section{Cuidados de anestesiología y reanimación para mantener las funciones vitales en pacientes donantes de órganos}

Vol. 3, núm. 3., (2019)

Alejandra de Jesús Álvarez Vásquez; Fanny Lissette Romero Loor; Fernanda Gisell García

Reyes; Mario Xavier Meza Cevallos; Karla Julen Vera Mendoza; Gema Margarita Reyes Ávila

un ritmo cardíaco objetivo de $\backslash 100$ latidos min-1 y / o un ritmo respiratorio de $\backslash 20$ respiraciones

min-1. Luego de la declaración de muerte, deben evitarse estrictamente las intervenciones que corren el riesgo de restablecer el flujo sanguíneo cerebral. Estos pueden incluir resucitación cardiopulmonar, derivación cardiopulmonar, oxigenación por membrana extracorpórea y / o ventilación mecánica con oxígeno para fines de recuperación pulmonar.

\section{Trasplante pulmonar}

Si se planea la adquisición de pulmón, se necesita un anestesiólogo para reintubar la tráquea del paciente después de la declaración de la muerte. Se debe administrar una sola maniobra de reclutamiento y los pulmones deben mantenerse abiertos con una presión positiva continua en la vía aérea de $10 \mathrm{~cm} \mathrm{H2O}$. Debido a que la ventilación con oxigenación puede llevar a un restablecimiento del suministro de oxígeno cerebral, la ventilación mecánica no debe iniciarse hasta que se haya aislado la circulación cerebral a través de una pinza cruzada a través de los vasos del arco aórtico. Una vez que se reinicie la ventilación mecánica, deben 6-8 mg kg-1 con 8-10cm H2O PEEP para disminuir el riesgo de lesión pulmonar aguda.

\section{Trasplante de hígado}

Aunque se han logrado resultados favorables con el uso de injertos de hígado DCD, ha habido una mayor tasa de complicaciones biliares asociadas con estos órganos, y por lo tanto, se considera que representan una opción de mayor riesgo para el receptor. Factores como el peso del paciente, el tiempo total de isquemia y la edad del donante pueden afectar el resultado del receptor. 


\section{Cuidados de anestesiología y reanimación para mantener las funciones vitales \\ en pacientes donantes de órganos}

Vol. 3, núm. 3., (2019)

Alejandra de Jesús Álvarez Vásquez; Fanny Lissette Romero Loor; Fernanda Gisell García Reyes; Mario Xavier Meza Cevallos; Karla Julen Vera Mendoza; Gema Margarita Reyes Ávila

Específicamente, el peso del donante [100 kg, el tiempo total de isquemia [nueve horas y la edad del donante [50 años] se han asociado con una mayor incidencia de colangiopatía isquémica.

\section{Trasplante de riñón}

Múltiples estudios han mostrado resultados similares en receptores que reciben un riñón de donantes con DCD o DBD. Al igual que con los donantes con DBD, debe evitarse un balance de líquidos negativo durante la obtención de órganos por DCD.

\section{Transplante de corazón}

Al igual que con otros órganos, los NHBD representan una fuente creciente de donación de órganos sólidos; sin embargo, el corazón puede ser el más sensible al tiempo de isquemia caliente, con 30 min considerado como un límite aceptable.45 El uso de cardioplejía puede ofrecer alguna promesa de un trasplante de corazón exitoso en el contexto de DCD, pero se necesita más investigación. (Abdelnour \& Rieke, 2009).

\section{Conclusiones.}

Los anestesiólogos desempeñan un papel vital en el manejo de los donantes de órganos, y la atención intraoperatoria puede afectar el resultado del órgano receptor. La donación después de las operaciones de adquisición de la muerte cerebral requiere un manejo intraoperatorio altamente específico por parte de un anestesiólogo. Para controlar el donante con muerte cerebral que palpita el corazón, el anestesiólogo debe incorporar el conocimiento de los efectos de la muerte cerebral en cada sistema orgánico, así como los efectos de las medidas preoperatorias que el donante 


\section{Cuidados de anestesiología y reanimación para mantener las funciones vitales en pacientes donantes de órganos}

Vol. 3, núm. 3., (2019)

Alejandra de Jesús Álvarez Vásquez; Fanny Lissette Romero Loor; Fernanda Gisell García

Reyes; Mario Xavier Meza Cevallos; Karla Julen Vera Mendoza; Gema Margarita Reyes Ávila

requiere en la UCI. También es importante saber qué órganos se van a adquirir para que se puedan establecer objetivos específicos y se puedan implementar estrategias (por ejemplo, ventilación protectora del pulmón o control glucémico intraoperatorio) para optimizar el resultado del donante. El éxito del anestesiólogo en la optimización fisiológica del donante con muerte cerebral puede determinar el resultado del órgano receptor. En el caso de la DCD, la intervención directa de un anestesista es necesaria para los donantes de pulmón. Además, pueden ayudar a garantizar que se preste la más alta calidad y la atención más adecuada a los donantes que no están latiendo del corazón al trabajar para establecer protocolos en sus hospitales para DCD que maximicen el número de órganos disponibles con la mejor posibilidad de injertos a largo plazo viabilidad.

\section{Bibliografía.}

Abdelnour, T., \& Rieke, S. (2009). Relationship of hormonal resuscitation therapy and central venous pressure on increasing organs for transplant. . J Heart Lung Transplant, 480-485.

Audibert, G., Charpentier, C., \& Seguin-Devaux, C. (2006). Improvement of donor myocardial function after treatment of autonomic storm during brain death. . Transplantation, 10311036.

Belzberg, H., Shoemaker, W., \& Wo, C. (2007). Hemodynamic and oxygen transport patterns after head trauma and brain death: implications for management of the organ donor. $J$ Trauma.

Bratton, S., \& Davis, R. (1997). Acute lung injury in isolated traumatic brain injury. Neurosurgery, 707-712.

Chen, E., Bittner, H., Kendall, S., \& Van Trigt, P. (1996). Hormonal and hemodynamic changes in a validated animal model of brain death. Crit Care Med, 1352-1359. 


\section{Cuidados de anestesiología y reanimación para mantener las funciones vitales \\ en pacientes donantes de órganos}

Vol. 3, núm. 3., (2019)

Alejandra de Jesús Álvarez Vásquez; Fanny Lissette Romero Loor; Fernanda Gisell García Reyes; Mario Xavier Meza Cevallos; Karla Julen Vera Mendoza; Gema Margarita Reyes Ávila

Cittanova, M., Leblanc, I., Legendre, C., Mouquet, C., Riou, B., \& Coriat, P. (1996). Effect of hydroxyethylstarch in brain-dead kidney donors on renal function in kidney-transplant recipients. Lancet, 1620-2.

Conci, F., Procaccio, F., Arosio, M., \& Boselli, L. (1986). Viscero-somatic and viscero-visceral reflexes in brain death. J Neurol Neurosurg Psychiatry, 695-698.

Dhar, R., Cotton, C., \& Coleman, J. (2013). Comparison of high- and low-dose corticosteroid regimens for organ donor management. . J Crit Care, 111.

Dominguez-Gil, B., Delmonico, F., \& Shaheen, F. (2011). The critical pathway for deceased donation: reportable uniformity in the approach to deceased donation. Transpl Int, 373378.

Gelb, A., \& Robertson, K. (1990). Anaesthetic management of the brain dead for organ donation. Can J Anaesth , 806-12.

Novitzky, D., Cooper, D., \& Reichart, B. (1987). Hemodynamic and metabolic responses to hormonal therapy in brain-dead potential organ donors. Transplantation , 852-4.

Pennefather, S., Bullock, R., Mantle, D., \& Dark, J. (1995). Use of low dose arginine vasopressin to support brain-dead organ donors. . Transplantation, 58-62.

RD., T. (2003). Organ donation after cardiac death: what role for anesthesiologists? Anesthesiology, 599-600.

Sally, M., Ewing, T., \& Crutchfield, M. (2014). Determining optimal threshold for glucose control in organ donors after neurologic determination of death: a United Network for Organ Sharing Region 5 Donor Management Goals Workgroup prospective analysis. $J$ Trauma Acute Care Surg , 68-69. 
Cuidados de anestesiología y reanimación para mantener las funciones vitales en pacientes donantes de órganos

Vol. 3, núm. 3., (2019)

Alejandra de Jesús Álvarez Vásquez; Fanny Lissette Romero Loor; Fernanda Gisell García

Reyes; Mario Xavier Meza Cevallos; Karla Julen Vera Mendoza; Gema Margarita Reyes Ávila

Shemie, S., Ross, H., \& Pagliarello, J. (2006). Organ donor management in Canada: recommendations of the forum on Medical Management to Optimize Donor Organ Potential. CMAJ, 13-32.

Steinbrook, R. (2007). Organ donation after cardiac death. . N Engl J Med, 357.

Szabo, G. (2004). Physiologic changes after brain death. J Heart Lung Transplant, 223-226.

U.S. Department of Health \& Human Resources. (2012). Organ Procurement and Transplantation Network. . Obtenido de http://srtr. transplant.hrsa.gov/annual_reports/2012/Default.aspx

Wijdicks, E., Varelas, P., Gronseth, G., \& Greer, D. (2010). Evidence-based guideline update: determining brain death in adults: report of the Quality Standards Subcommittee of the American Academy of Neurology. American Academy of Neurology. , 74.

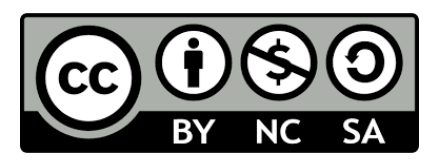

RECONOCIMIENTO-NOCOMERCIAL-COMPARTIRIGUAL

CC BY-NC-SA

ESTA LICENCIA PERMITE A OTROS ENTREMEZCLAR, AJUSTAR Y CONSTRUIR A PARTIR DE SU OBRA CON FINES NO COMERCIALES, SIEMPRE Y CUANDO LE RECONOZCAN LA AUTORÍA Y SUS NUEVAS CREACIONES ESTÉN BAJO UNA LICENCIA CON LOS MISMOS TÉRMINOS. 